\title{
Dynamic Bandwidth Allocation for Next-generation OFDMA-PON to Improve QoS
}

\author{
Yunfeng Peng \\ School of Compu. \& Commun. Engineering \\ Univ. of Sci \& Tech Beijing \\ Beijing, China \\ e-mail: Pengyf@ustb.edu.cn
}

\author{
Xiuping Guo \\ School of Economics \& Management \\ Southwest Jiaotong University \\ Chengdu, China \\ e-mail:maxgxp@gmail.com
}

\begin{abstract}
This work proposes a Dynamic Bandwidth Allocation (DBA) scheme for next-generation OFDMA-PON to accommodate wireless signals. We transform DBA into a multi-objective optimization problem, and Non-dominated Sorting Genetic Algorithm is applied to get best-fitted individuals for each Optical Network Unit (ONU) to achieve low delay and fairness.
\end{abstract}

Keywords-Passive Optical Networks; Dynamic Bandwidth Allocation ; Genetic Algorithm

\section{INTRODUCTION}

Passive Optical Network (PON) provides a point-tomultipoint solution to satisfy the increasing demand on capacity in access networks, i.e., between service provider central offices (COs) and customer sites. A PON consists of an optical line terminal (OLT) located at the provider $\mathrm{CO}$ and a number of optical network units (ONUs) at the customer premises [1].

Some candidates for next-generation PON stand out, including Time Division Multiple Access-based (TDMAbased) 10Gigbit-PON (10G-PON), 10Gigbit Ethernet-PON (10GE-PON) and Wavelength Division Multiplexing-PON (WDM-PON) [2], as well as Orthogonal Frequency Division Multiple Access-PON (OFDMA-PON) [3]. However, migration to these new technologies has raised a number of challenges.

Current commercial PONs adopts centralized medium access control (MAC) to govern downstream and upstream data among multiple ONUs. To arbitrate bandwidth allocation for upstream transmission, dynamically bandwidth allocation (DBA) algorithm is required.

The most outstanding DBA, Interleaved Polling With Adaptive Cycle Time (IPACT) [4], is noted as the founder of DBA design. To provide differentiated services and guaranteed QoS, Bandwidth Guaranteed Polling (BGP) mechanism [5] is proposed to equally divide bandwidth into several parts and be allocated to ONUs with higher priority according to service-level agreement (SLA). However, equal bandwidth division may result in data fragmentation, which leads to extra guard band, and decrease throughput and bandwidth utilization.

In order to increase bandwidth utilization and share optical wavelength elastically, dynamic wavelength and bandwidth allocation (DWBA) algorithm [6] is proposed, which assumes that each ONU can switch wavelength in upstream channel at millisecond level. However, it is expensive to switch wavelength at millisecond level in TDMA-PON. Orthogonal Frequency Division Multiplexing (OFDM) technology uses multiple carriers overlapped orthogonally in the frequency domain, resulting in higher spectral efficiency. Individual subcarriers can be allocated to different ONUs in OFDMA-PON, to achieve twodimensional time-frequency bandwidth map at much finer granularity compared with TDMA-PON and WDM-PON.

Ref. [7] proposed two MAC agreements, dynamic circuit transmission (DCT) and fixed burst transmission (FBT) for OFDMA-PON. DCT is only suitable for non-bursty traffic, while FBT does not provide satisfactory QoS performance since the scheduling algorithm is based on a simple roundrobin strategy. In [8], a set of subcarriers assigned to each "virtual PONs". However, this design works in pure TDMA manner and will not fit in architecture presented in [9].

We in this work propose a feasible and fast bandwidth allocation schemes for next-generation OFDMA-PON. We model the complex DBA into a multi-objective optimization problem with QoS of mean delay and fairness of standard deviation of mean delay among ONUs as two objectives. Non-dominated Sorting Genetic Algorithm (NSGA) [10,11] is used to solve the problem.

The remainder of this paper is organized as follows. In section II, we present the architecture of OFDMA-PON and elaborate on how the system works. In section III, we state the inherent DBA problem for the OFDMA-PON architecture. In section IV, we adopt NSGA to promote QoS and fairness among ONUs and delineate the proposed dynamic bandwidth allocation scheme. In section $\mathrm{V}$, algorithm performance is analyzed according to simulation results. In section VI, conclusions are remarked.

\section{OFDMA-PON ARCHITECTURE}

The bandwidth allocation design is based on the OFDMA-PON architecture shown in Fig.1, where Optical Line Terminal (OLT) is connected to multiple remote ONUs through the Optical Distribution Node (ODN). The ODN is composed of a set of optical circulators to direct signals among ONUs and OLT. 


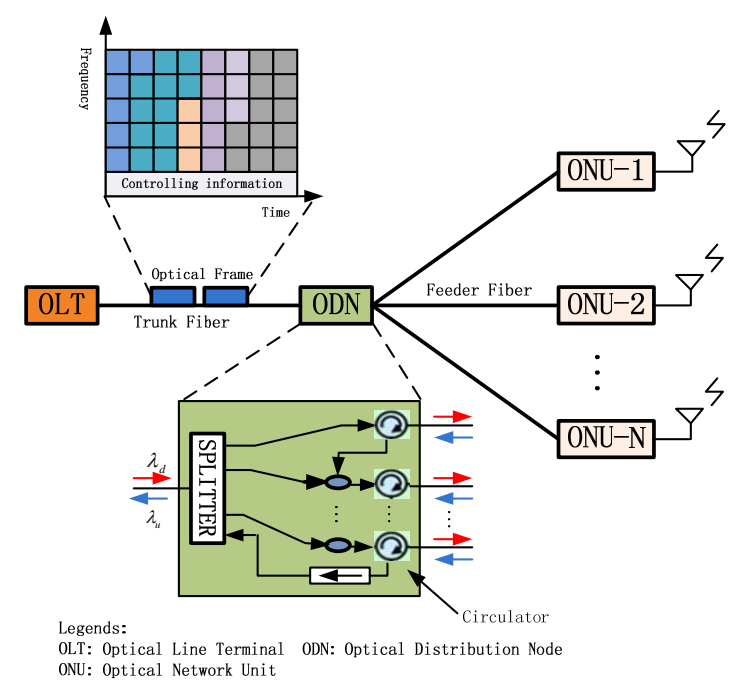

Figure 1. OFDMA-PON system architecture [9]

Compared with OFDMA-PON architecture proposed in [3]. This architecture is cost-effective since it requires only two wavelengths $\lambda_{\mathrm{d}}$ and $\lambda \mathrm{u}$, for downstream and upstream respectively. Additionally, it is able to alleviate Optical Beat Interference (OBI) in the trunk fiber thus to maintain the signal quality of transmission.

However, works about bandwidth allocation scheme for this architecture are scarce.

\section{NSGA BASED DBA ALGORITHM}

We firstly define two factors, i.e., cycle and bandwidth unit to describe our DBA design. One cycle is the time scale of a round of upstream transmission by all ONUs. The bandwidth unit is one OFDM sub-carrier, which is the minimal resource one ONU can get in a certain cycle. The downstream ONUs will suffer more time delay without careful designing.

Two parameters are introduced, i.e., Transmit Quota Capacity (TQC) and Transmit Quota (TQ). TQC is volume of virtual buffer for each ONU to limit maximum burst length. TQ is the access rate for ONUs to send its packets in each cycle. The higher TQC is, the larger burst of packets an ONU can transmit in a cycle. The higher TQ is, the greater mean number of packets an ONU can transmit. Below is illustration of TQC and TQ cooperation.

Step 1: OLT provides totally $\mathrm{K}$ sub-carriers available in a certain cycle. Upstream transmission starts from ONU-1.

Step 2: For ( $\mathrm{i}=1, \mathrm{i}<=\mathrm{N}, \mathrm{i}++)$, ONU-i extracts (TQ', TQC') information encapsulated at controlling sub-channel and update. $\mathrm{TQ}_{\mathrm{i}}=\mathrm{TQ}_{\mathrm{i}}+\mathrm{TQ}_{\mathrm{i}}{ }^{\prime} . \quad \mathrm{TQC}_{\mathrm{i}}=\mathrm{TQC}_{\mathrm{i}}+\mathrm{TQC}_{\mathrm{i}}{ }^{\prime}$. If $\left(\mathrm{TQ}_{\mathrm{i}}>\mathrm{TQC}_{\mathrm{i}}\right)$, remove surplus transmit quota until $\mathrm{TQ}_{\mathrm{i}}=\mathrm{TQC}_{\mathrm{i}}$.

Where dotted $\mathrm{TQ}_{\mathrm{i}}{ }^{\prime}$ and $\mathrm{TQC}_{\mathrm{i}}{ }^{\prime}$ represent the newly endowed transmit quota and capacity by OLT, respectively. While $\mathrm{TQ}_{\mathrm{i}}$ and $\mathrm{TQC}_{\mathrm{i}}$ represent the remaining transmit quota and capacity after previous cycle.

Step 3: ONU-i starts to transmit. $\mathrm{RSC}_{\mathrm{i}}$ represents number of Remaining Sub-carriers, obviously, $\mathrm{RSC}_{1}=\mathrm{K}$. Pkt $\mathrm{i}$ represents number of packets waiting for transmission in
ONU-i. Hence, number of packets needing to be sent is determined by expression $\min \left\{T Q_{i}, R S C_{i}, p k t_{-} i\right\}$. Then do

$$
\begin{gathered}
R S C_{i+1}=K-\sum_{j=1}^{i} \min \left\{T Q_{j}, R S C_{j}, p k t_{-} j\right\} \\
T Q_{i}=T Q_{i}-\min \left\{T Q_{i}, R S C_{i}, p k t_{-} i\right\}
\end{gathered},
$$

Step 4: when all the subcarriers are used up or all ONUs finish transmission, ONU-N send all packets upstream to OLT, and then cycle ends.

To assess QoS, we define delay as the time duration one packet waits in ONU. If one packet arrives at a certain ONU at cycle $T_{1}$, and is transmitted at cycle $T_{2}$, the delay of the packet is $T_{2}-T_{1}$. Hence we have the mean delay for ONU-i:

$$
\text { mean_delay }(i)=\frac{\sum_{j=1}^{\text {\#of packets_sent }} \operatorname{delay}(j)}{\sum_{\text {all }} \# \text { of packets_sent }}
$$

We use mean delay of each ONU to measure QoS and the standard deviation of mean delay among all ONUs to measure fairness. While monitoring network traffic, TQ and TQC may be adjusted to let ONUs share mean delay as small and equal as possible, to achieve better QoS and fairness. According to this design, when upstream ONUs have large number of packets to be send, TQC take the role of limiting burst packets number. Once the quota is used up, TQ has to be accumulated. We set higher TQ or TQC on downstream ONUs to increase their chances of sending packets, so to reduce mean delay.

In this architecture, as illustrated in Fig.2, upstream ONUs has no knowledge of bandwidth requirement from downstream ONUs. Therefore, the direct way is to compare mean delay between two adjacent ONUs. For instance, if mean delay(2) for ONU-2 is less than mean delay(1) for ONU-1, then we should decrease TQ or TBS of ONU-2 until mean-delay(1) is equal to mean-delay(2). By this way, mean delay of downstream ONUs can be gradually equalized to mean_delay(1) and fairness can be achieved at the same time. However, this manner is not feasible because it takes much time to manually adjust (TQ, TQC).

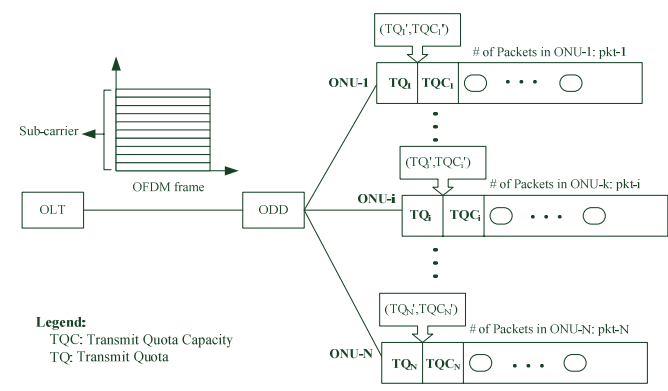

Figure.2. Illustration of upstream transmission in a certain cycle

Proposed by Deb, K in [10, 11], Non-dominated Sorting Genetic Algorithm (NSGA) is one method to solve multiobjective optimization problem. In our DBA design, we firstly map TQ and TQC from decision space to objective 
space, where NSGA works. To be noted, mean delay of ONU-i is entirely dependent on TQ and TQC of ONU-i itself, as well as TQs and TQCs of all preceding ONUs. Here, mapping function, also named objective function can be expressed as a function of parameters TQ and TQC, i.e.,

$$
\text { mean_delay }(i)=f_{i}\left(T Q_{1}, \ldots, T Q_{i-1}, T Q_{i} ; T Q C_{1}, \ldots, T Q C_{i-1}, T Q C_{i}\right)
$$

In our DBA design, TQ and TQC of each ONU are taken as genes to run NSGA. Via NSGA computation, new bestfitted genes are found out and broadcasted to all ONUs, until specified generations are done or no more improvement can be achieved. The detailed NSGA based DBA is demonstrated in Fig.3.

In NSGA, two fitness functions on QoS and fairness needed to meet our DBA requirements.

To measure QoS, we put forward the fitness function 1 as:

$$
f 1=\frac{\sum_{i=1}^{N} \mu_{i}}{N}
$$

$\mathrm{N}$ is number of ONU, ${ }^{\mu}$ is the mean delay of ONU-i. Fitness function 1 is the average mean-delay of all ONUs, the smaller its numerical value is, the less delay ONUs suffer, and the larger network throughput will be. To measure fairness, we put forward the fitness function 2 as:

$$
f 2=\frac{\sqrt{\sum_{i=1}^{22}\left(\mu_{i}-\overline{\mu_{0}}\right)^{2}+\sum_{j=23}^{32}(j-22)\left(\mu_{j}-\overline{\mu_{0}}\right)^{2}}}{\overline{\mu_{0}}} .
$$

$\overline{\mu_{10}}$ represents the mean delay of the first 10 upstream ONUs. Fitness function 2 is standard deviation of mean delay among all ONUs and the first 10 upstream ONUs. The mean delay of first 10 upstream ONUs is equally the same, and we add weight to last 10 ONUs to handle the mean delay unfairness. The smaller its numerical value is, the greater chances the curve will be, which means greater fairness.

\section{SIMULATION AND ANALYSIS}

The simulation investigates the mean delay and delay fairness with respect to various TQC setting. The reason for using TQC instead of TQ is two-fold. First, TQC is an integer, which is simple to adjust. In addition, as we analyzed above, system performance is as sensitive to TQC settings as to TQ. In the simulation, the PON system has $\mathrm{N}=32$ ONUs operating at $10 \mathrm{~Gb} / \mathrm{s}$, and all ONUs share a total of 512 OFDM data sub-channel slots. Each OFDM slot is 16 bits long.

In the first simulation, we set traffic load to 0.9 , burst to 8.0, fix TQ to16.0, and then adjust parameter TQC, so that all ONUs are with the same mean delay. On the simulation result shows in Fig.4, we find two problems, (1) It has limitation to manually adjust TQC on fixed TQ, especially when mean delay has dropped down to $5 \mathrm{~ms}$ or so, because the range of TQC is too distributive to be controlled. (2) It is

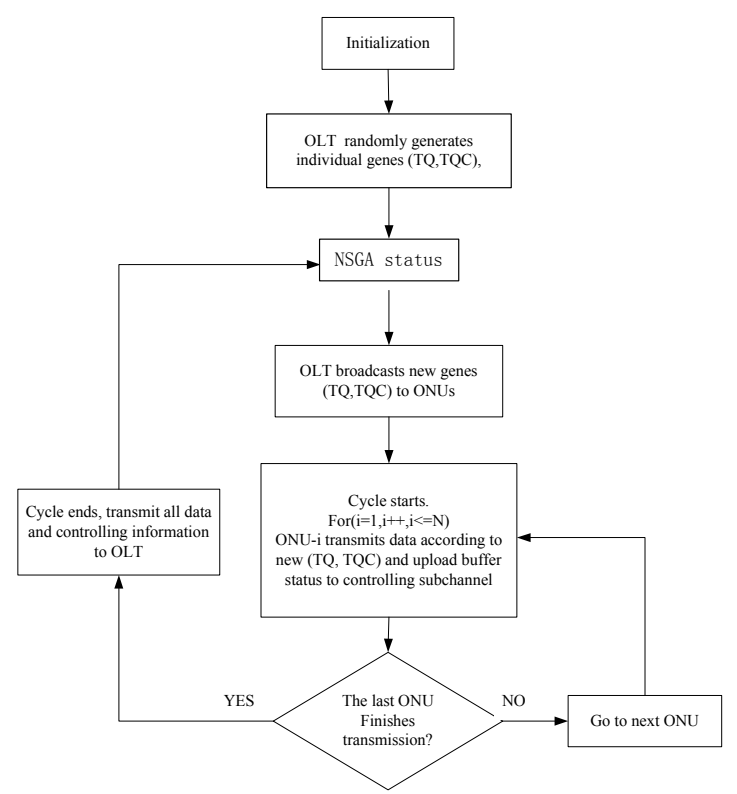

Figure.3. NSGA Based DBA Algorithm

even impossible to dynamically allocate OFDM-frames on varying traffic.

In the second simulation, we apply NAGA to find out best fitted (TQ, TQC) settings. Fig.4 illustrates that the curve is exponential shape alike, so we express TQC using exponential function. Input variables are $\mathrm{TQ}=16.0$ for all the ONUs,

$$
T Q C_{i}=\exp (a * i+b)+\exp (c * i+d)+e
$$

Other simulation environment keeps the same as in first simulation.

Fig. 5 is pareto front of the generated 60 pairs (f1, f2) combination. As it can be observed, trade-off exists between mean delay and ONU fairness. The larger mean delay is, the greater fairness ONU achieves, and vise versa.

We select three typical ( $1, f 2)$ combination as illustrated in Fig.6, which indicates that mean delay for upstream ONUs drops at end ONUs. The reasons behind is that we add increasing weights to last 10 ONUs to reduce mean delay.

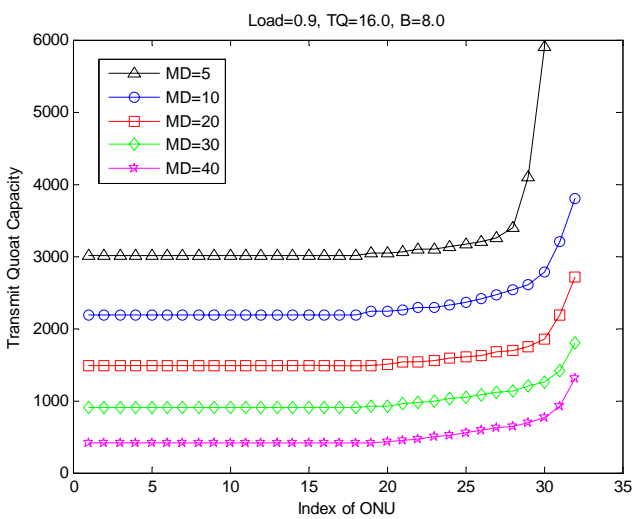

Figure.4. TQ fixed, manually adjust TQC 


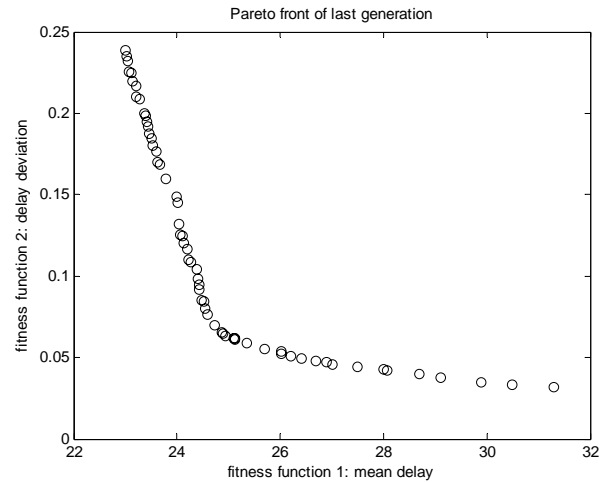

Figure.5. Pareto front of exp. TQC

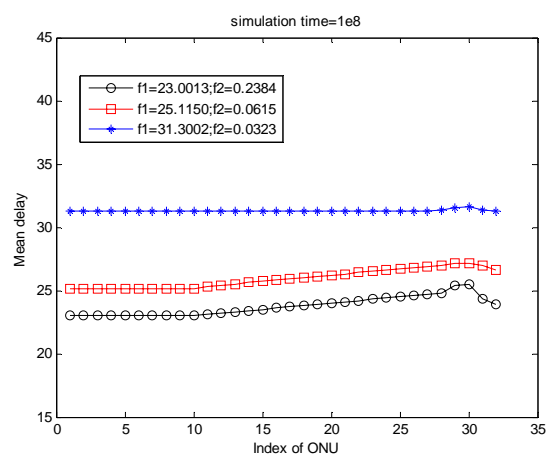

Figure.6. Three typical (fi,f2) combination of exp. TQC results

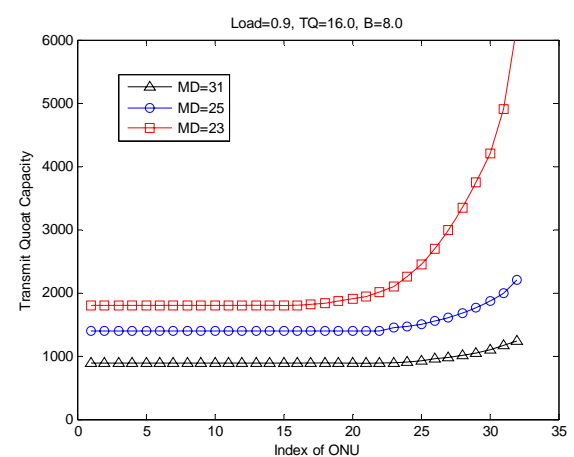

Figure.7. NSGA based TQC settings to achieve QoS and ONU fairness

Fig.7 illustrates the impact of TQC on mean delay and ONU fairness as TQC being around 2000. The mean delay can be $23 \mathrm{~ms}$, but there exists unfairness among ONUS. Especially for downstream ONUs, too many TQC is used to satisfy the mean delay. As TQC decreased, mean delay increases to $31 \mathrm{~ms}$, which is still tolerable to communication services, so that objectives of the DBA design, QoS and fairness, are achieved simultaneously.

\section{CONCLUSIONS}

In this paper, we propose an optimal and efficient dynamic bandwidth allocation (DBA) for next-generation OFDMA-PON. In the DBA, we apply Non-dominated Sorting Genetic Algorithm (NSGA) to design a TQ-TQC based DBA scheme to dynamically return the optimal TQ and TQC values for all ONUs. From the simulation results, the DBA design can satisfy the required time delay and ONU fairness under varying traffic conditions.

\section{ACKNOWLEDGMENT}

This work is supported by National Natural Science Foundation of China (No.61071101), Program for New Century Excellent Talents in University (NCET-11-0709), and Open Foundation of State Key Laboratory of Advanced Optical Communication Systems and Networks, Shanghai Jiao Tong University (2013GZKF031311).

\section{REFERENCES}

[1] Jiajia Chen ; Ahmed, J. ; Wosinska, L. ; Mukherjee, B. ; "A comparison of dynamic bandwidth allocation for EPON, GPON, and next-generation TDM PON. " Communications Magazine, IEEE., vol. 47, no. 3, Mar. 2009, pp. 40-48.

[2] Effenberger, F.; Mukai, H.; Soojin Park; Pfeiffer, T.; "Nextgeneration PON-part II: Candidate systems for next-generation PON." Communications Magazine, IEEE vol. 47 , no. 11, Nov. 2009 , pp. 50 $-57$.

[3] Dayou Qian; Cvijetic, N.; Junqiang Hu; Ting Wang.; "Optical OFDM transmission in metro/access networks." Optical Fiber Communication. 2009, pp. 1-3.

[4] Kramer G, Mukherjee B, Pesavento G, "IPACT: A Dynamic Protocol for an Ethernet PON (EPON)," IEEE Communications Magazine, vol.40.no.2,Feb.2002,pp.74-80.

[5] Ma M., Zhu Y., Cheng T.H. , "A bandwidth guaranteed polling MAC protocol for Ethernet passive optical networks,"INFOCOM 2003,vol.1.Apr.2003.

[6] Dhaini A.R., Assi C.M., Maier, M., Shami, A."Dynamic Wavelength and Bandwidth Allocation in Hybrid TDM/WDM EPON Networks,"IEEE journal of Lightwave technology, vol.25, no.25, Jan.2007,pp.277-286.

[7] Wei Wei;Ting Wang;Dayou Qian;Junqiang Hu;"MAC Protocols for Optical Orthogonal Frequency Division Multiple Access (OFDMA)based Passive Optical Networks,"IEEE/OSA OFC,2008.

[8] Wei Wei et all.; "PONIARD: A Programmable Optical Networking Infrastructure for Advanced Research and Development of Future Internet." J.Lightw.Technol.,vol. 27 ,no. 3, Feb. 2009, pp. 233-242.

[9] Yu-min Lin; Po-lung Tien; "Next-generation OFDMA-based passive opticalnetwork architecture supporting radio-over-fiber." IEEE Journal on Selected Areas in Comm., vol. 28, no. 6, Aug. 2010, pp. 791-799.

[10] Deb K., Pratap A., Agarwal S., and Meyarivan T, "A fast and Elitist Multi-Objective Genetic Algorithm-NSGA-II," IEEE Transactions on Evolutionary Computation, Vol. 6, NO. 2, Apr. 2002, pp.182-197.

[11] Deb. K. Multi-Objective Optimization using Evolutionary Algorithms, John Wiley \& Sons, first edition, March 2009. 